\title{
Ultrastructure of Flagellated Lambda Symbionts in Paramecium aurelia
}

\author{
By A. JURAND AND LOUISE B. PREER \\ Institute of Animal Genetics, Edinburgh, Scotland \\ (Accepted for publication 24 July 1968) \\ SUMMARY
}

The symbionts called 'lambda' present in the cytoplasm of killer stocks 239 (syngen 4) and 299 (syngen 8 ) of Paramecium aurelia have been investigated. Observations with the electron microscope of ultrathin sections and of negatively stained material revealed that these symbionts have peritrichous flagellation. The ultrastructures of symbionts from both stocks were identical and were those of flagellated bacteria.

\section{INTRODUCTION}

Four stocks of Paramecium aurelia bear cytoplasmic symbionts known as lambda: stock 239 (syngen 4), stocks, 216, 229 and 299 (syngen 8). They were described by Schneller (1957, 1961, 1962), Schneller, Sonneborn \& Mueller (1959) and Sonneborn (1950, 1959, 1965). These stocks are referred to as rapid lysis killers, since sensitive paramecia are lysed within $20 \mathrm{~min}$. after being exposed to them. Paramecia of syngen 3 are especially sensitive to the action of all lambda-bearing killers. Lambda particles are much larger than most of the other symbionts which have been described for $P$. aurelia. Van Wagtendonk, Clark \& Godoy (I963) reported that it was possible to grow lambda particles in cell-free cultures in an axenic medium. There has been little electron microscopy of lambda reported. Sonneborn (1965) published one micrograph of this symbiont. The present paper reports investigations on lambda of $P$. aurelia stocks 239 and 299 by use of phase-contrast and electron microscopy.

\section{METHODS}

Both stocks of Paramecium were grown in lettuce medium previously inoculated with Aerobacter aerogenes (Sonneborn, 1950). Lambda particles outside the host cytoplasm were observed with a bright phase-contrast microscope (American Optical Co.) in animals freshly crushed between a slide and coverslip. To observe the symbionts in situ two methods were used. In the first, whole paramecia were observed with a dark phase-contrast microscope (Carl Zeiss), using paramecia treated with lactoorcein according to the method of Beale \& Jurand (I966). In the second, I $\mu$ Araldite sections were mounted on slides, stained with toluidine blue and observed with ordinary bright-field microscopy.

The ultrastructure of lambda symbionts was investigated by examination of ultrathin sections and of negatively stained isolated symbionts with the A.E.I. 6 electron microscope. For ultrathin sectioning mass cultures of each stock were fixed in one of two ways. In the first method the paramecia were concentrated by centrifugation, 
and the supernatant culture medium replaced with a modified Palade (1952) fixative containing $15 \mathrm{mg}$. sucrose and $\mathrm{O} \cdot \mathrm{I} \mathrm{mg}$. calcium chloride $/ \mathrm{ml}$. fixative. Fixation was for $30 \mathrm{~min}$. at room temperature $\left(20^{\circ}\right)$ or at $37.5^{\circ}$, and during this time the suspension of paramecia was gently stirred every few minutes. After fixation the paramecia were concentrated by centrifugation and dehydrated with aqueous $35,70,90$, and $100 \%$ ethanol, and embedded in Araldite on a slow rotary shaker (Jurand \& Ireland, 1965) for I hr. Some preparations were made by using I,2-epoxy-propane + Araldite before embedding in Araldite.

The second method of fixation used a $30 \%(\mathrm{w} / \mathrm{v})$ solution of osmium tetroxide in carbon tetrachloride (Afzelius, 1962). The osmium tetroxide solution was prepared just before use, and $0.3 \mathrm{ml}$. was added to a concentrated suspension of paramecia in the culture medium $(0.6$ to $0.8 \mathrm{ml}$.). The mixture was left for $20 \mathrm{~min}$. at room temperature and the test-tube gently shaken frequently. Carbon tetrachloride and the culture medium do not mix, and fixation takes place in the culture medium into which osmium tetroxide penetrates very readily from the carbon tetrachloride layer. After fixation, $70 \%(\mathrm{v} / \mathrm{v})$ ethanol in water was slowly added and the mixture gently shaken until the two layers united. The mixture was then centrifuged, and the supernatant fluid discarded and replaced by $70 \%(\mathrm{v} / \mathrm{v})$ ethanol in water. Further dehydration and embedding was the same as in the first method. This second method of fixation is particularly suitable for preservation of various cytoplasmic symbionts in different stocks of Paramecium aurelia. For example, it was found to prevent shrinkage and preserve ultrastructure of refractile bodies and virus-like particles in kappa of stocks 7 and 562 (Preer \& Jurand, 1968). On the other hand, the host cell organelles are usually well preserved with the modified Palade fixative (1952).

Partially purified lambda symbionts were prepared for negative staining as follows. A homogenate of $0.3 \mathrm{ml}$. of paramecia of stock 239 in $10 \mathrm{ml}$. of Dryl (I959) solution was diluted with an equal volume of o.or M-sodium phosphate buffer $(\mathrm{pH} 7)$ and passed through a cellulose column $12 \mathrm{~mm}$. in diameter and $50 \mathrm{~mm}$. high. The column was prepared by suspending 2 g. Whatman Ashless Filter Paper Pulp in distilled water and then washing with phosphate buffer. Examination with the bright phase-contrast microscope indicated that the turbid wash from the column was free from trichocysts and fragments of the cell body wall. The large lambda particles were separated from the lipid droplets, mitochondria and fragments of cilia in the turbid wash by a light centrifugation of $1000 \mathrm{~g}$ for $5 \mathrm{~min}$. About $90 \%$ of the particles in the pellet were lambda, with a few bacteria and mitochondria. A drop of the preparation was mixed with a drop of $5 \%(\mathrm{w} / \mathrm{v})$ phosphotungstic acid $(\mathrm{pH} 7)$ and examined with the electron microscope. Bacterial contaminants of two kinds were present. Their shape and small size made it possible to distinguish them readily from the numerous and much larger lambda particles.

To decrease to a minimum the number of bacteria present in the final preparation of isolated lambda particles and to aid the identification of the few bacteria which were present, a modified procedure was followed for the isolation of stock 299 lambda particles. The culture of paramecia was concentrated by centrifugation and the supernatant fluid which contained the bacteria was retained for later use as described below. The precipitate of packed paramecia was resuspended in $25 \%(\mathrm{w} / \mathrm{v})$ sterile yeast extract (Difco) at a concentration of about $0.1 \mathrm{ml}$. packed organisms in $100 \mathrm{ml}$. yeast extract solution. The paramecia were allowed to remain at room temperature 
for 30 to $60 \mathrm{~min}$., then filtered through cheesecloth and centrifuged. This process was repeated twice. Food vacuoles of paramecia treated in this way were seen to have no bacteria in them. These paramecia were then treated according to the procedure that was used to isolate lambda particles from stock 239. The lambda particles of stock 299 collected in the turbid wash from the cellulose column had very few contaminating bacteria among them. This preparation was examined with bright phase-contrast and electron microscopy. To establish a clear picture of the bacterial contaminants present, the original culture medium in which the paramecia had been grown, and from which they had been removed, was centrifuged at $8000 \mathrm{~g}$ for $5 \mathrm{~min}$. The concentrated bacteria of the pellet were examined in bright phase-contrast and electron microscopes. This made possible the recognition of any bacteria, however few, which were in the preparations of isolated lambda particles.

\section{RESULTS}

Lambda symbionts in Paramecium aurelia stocks 239 and 299 were distributed throughout the host cytoplasm (Pl. I, fig. I to 4). Usually there were more lambda particles present in stock 239 organisms than in stock 299 organisms grown under the same conditions. The symbionts were Gram negative bacterium-like particles, 2 to $4 \mu$ long and about $0.5 \mu$ wide, and were very similar in the two stocks. Lambda of crushed paramecia of stock 299 are shown as seen in the bright phase-contrast microscope in Pl. I, fig. 5. A partially-purified preparation of lambda particles of stock 239 obtained from the cellulose column is shown in Pl. I, fig. 6.

Examination of ultrathin sections of paramecia with the electron microscope revealed that lambda symbionts in both stocks were flagellated. The flagella were fairly evenly distributed over the entire surface of the symbiont, and the general ultrastructure of the flagella in both stocks was identical. In situ, the lambda particles, together with flagella, were usually enclosed within elongated vacuoles delimited from the host cytoplasm by an interface boundary. Two or three symbionts might be enclosed in a single vacuole (Pl. I, fig. 7,8 ).

The presence of numerous flagella was confirmed by examining with the electron microscope negatively stained preparations of partially purified lambda ( $\mathrm{Pl}$. 2, fig. 9, Io). It is difficult to give an approximate figure for the number of flagella present on one symbiont. In sectioned material the flagella were visible only as fragments, and in negatively stained preparations it was quite possible that some of the flagella may have been lost because of mechanical breakage during purification. It was estimated that there might be at least 100 flagella on the entire surface of one lambda symbiont. This estimate was made from Pl. 2, fig. II, where there are a number of flagella sectioned transversely.

In ultra-thin sections the diameter of the flagella appeared to be about $170 \AA$. The ultrastructure was much less granular than in negatively stained preparations and occasionally showed transverse striations. In negatively stained preparations the measurements are uncertain because of the flattening of flagella during desiccation and because it is difficult to estimate the extent to which the negative stain penetrated the flagella. In these preparations the flagella very frequently assumed a characteristic sinusoidal form and were up to $5 \mu$ long (Pl. 3, fig. I2). Their ultrastructure in negatively stained preparations was granular and appeared as two rows of linearly arranged 
electron-dense subunits extending along the length of the flagellum symmetrically on both sides at about one quarter of its diameter (Pl. 3, fig. I3).

The lambda symbionts were enclosed in a cell wall consisting of two unit membranes. Inside the symbionts there were tightly-packed dense granules about $120 \AA$ in diameter. The distribution of these granules, although dense, was not very uniform. There were less dense areas without granules occupied by elongated fibrillar structures about $500 \AA$ long and $200 \AA$ wide (Pl. 3, fig. I4).

To investigate whether flagella confer mobility on the lambda particles, paramecia were crushed between a slide and coverslip and observed with a bright phase-contrast microscope. In these conditions the lambda particles often showed a limited 'wiggling' motion, quite different from the Brownian movement. However, no clear swimming movement was observed.

\section{DISCUSSION}

Cytoplasmic symbionts in paramecia have long been regarded as bacterium-like. This idea is further supported by the present work which shows that lambda particles have several features which are characteristic of bacteria. First, the filamentous appendages of lambda resemble bacterial flagella. They are more like flagella than other similar bacterial structures such as pili (fimbriae). This conclusion is reached from a consideration of the length, diameter, surface distribution and especially the sinusoidal form of these appendages in negatively stained preparations. The length and diameter are within the ranges for these structures in bacteria (Newton \& Kerridge, 1965). The more or less uniform distribution over the surface of the lambda particle is peritrichous flagellation, which is very common in bacteria (Leifson, 1960). Moreover, after drying the sinusoidal form is typical of bacterial flagella (Stocker, 1956), whereas pili are always straight (Brinton, I965). The flagella of lambda particles are strikingly similar in shape to most of the flagella of bacteria shown in the paper of Houwink \& Iterson (1950), which is a most informative work on this subject. The ultrastructure of lambda flagella is virtually identical with that of bacterial flagella, e.g. in Proteus vulgaris (Abram, Koffler \& Vatter, I965). Secondly, lambda particles give a negative reaction with the Gram reagents and the ultrastructure of the wall is very like that of Gramnegative bacteria such as Escherichia coli, Spirillum species and others (Murray, Steed \& Elson, 1965; van Iterson, 1965). The two thin double-unit membranes observed in lambda are characteristic of Gram-negative bacteria. Thirdly, the ultrastructure of the intracellular content of lambda particles is similar to that of bacterial cytoplasm. The tightly packed dense granules in lambda particles resemble bacterial ribosomes, and the elongated fibrillar structures present in the less dense areas are similar to bacterial nucleoids (van Iterson, 1965; Fuhs, 1965). Altogether, filamentous appendages which resemble bacterial flagella, the ultrastructure of the cell wall which is very similar to that of Gram-negative bacteria, and the ultrastructure of the cytoplasm which is similar to the cytoplasm of bacteria, all make it evident that a lambda particle may well be regarded as a prokaryotic unicellular organism, a Gramnegative bacterium.

\section{ADDENDUM}

After this lpaper was sent for publication we obtained the two remaining stocks known to contain lambda particles, stocks 216 and 229 (syngen 8), and also stock II4 
(syngen 2) which contains sigma particles (Sonneborn, Mueller \& Schneller, 1959). These three stocks, like stocks 239 and 299, are rapid lysis killers.

The lambda particles of stock 216 and 229, and sigma of stock I 14 were investigated by using the same methods as described in the main part of this paper. The lambda particles of stocks 216 and 229 appeared to be very similar to those of stocks 239 and 299. The measurements of the lambda particles, the peritrichous flagellation and the ultrastructure of the flagella in all four lambda stocks were virtually identical. Two differences were noted: in stock 229 the diameter of the flagella was the greatest of all of the lambda particles, measuring about $240 \AA$, whereas in stock 216 it was $200 \AA$, in both cases measured in sections; secondly, in stock 216 , at least in the culture investigated, lambda particles were often enclosed in larger vacuoles containing several (up to 12) particles, whereas in the other stocks the symbionts usually occurred singly, or in pairs, in one vacuole. Sigma particles of stock I I 4 were of the same diameter as lambda particles but were Io to I $5 \mu$ long and spirally curved. Flagella of sigma particles were very similar in ultrastructure and in their peritrichous distribution to those of lambda particles. Sigma particles were enclosed in vacuoles singly or in pairs.

In summary, all of the stocks known to kill by rapid lysis, i.e., the lambda-bearing stocks 216, 229, 239 and 299 and the sigma-bearing stock II4, contain particles which are morphologically similar. Observations with the electron microscope indicate that lambda and sigma particles are flagellated bacteria.

We wish to thank the National Science Foundation of the United States and the Phi Beta Psi Sorority for research grants to one of us (L.B.P.). We also wish to thank Professor G. H. Beale, F.R.S., and Professor J. R. Preer, Jun. for their interest and encouragement, and Dr J. F. Wilkinson of the Edinburgh School of Agriculture for discussions of relevant microbiological problems.

\section{REFERENCES}

Abram, D., Koffler, H. \& VATTER, A. E. (1965). Basal structure and attachment of flagella in cells of Proteus vulgaris. J. Bact. 90, 1337.

AfZelius, B. A. (1962). Chemical fixatives for electron microscopy. In Interpretation of Ultrastructure, p. I. Ed. by R. J. C. Harris. New York and London: Academic Press.

BeAle, G. H. \& JuRAND, A. (1966). Three different types of mate-killer (m $\mu$ ) particle in Paramecium aurelia (syngen I). J. Cell Sci. I, 3I.

Brinton, C. C. (1965). The structure, function, synthesis and genetic control of bacterial pili and molecular model for DNA and RNA transport in Gram-negative bacteria. Trans. N.Y. Acad. Sci. 27, 1003.

DRYL, S. (1959). Antigenic transformation in Paramecium aurelia after homologous antiserum treatment during autogamy and conjugation. J. Protozool. 6, Suppl. 25.

FuHs, G. W. (1965). Symposium on the fine structure and replication of bacteria and their parts. $\mathbf{I}$. Fine structure and replication of bacterial nucleoids. Bact. Rev. 29, 277.

Houwink, A. L. \& van Iterson, W. (1950). Electron microscopical observations on bacterial cytology. II. A study on flagellation. Biochim. biophys. Acta 5, 10.

Jurand, A. \& Ireland, M. J. (1965). A slow rotary shaker for embedding in viscous media. Stain Technol. 40, 233.

LEIFSON, E. (1960). Atlas of Bacterial Flagellation. New York \& London: Academic Press.

MurRay, R. G. E., Steed, P. \& Elson, H. E. (1965). The location of the mucopeptide in sections of the cell wall of Escherichia coli and other Gram-negative bacteria. Can. J. Microbiol. 11, 547. 


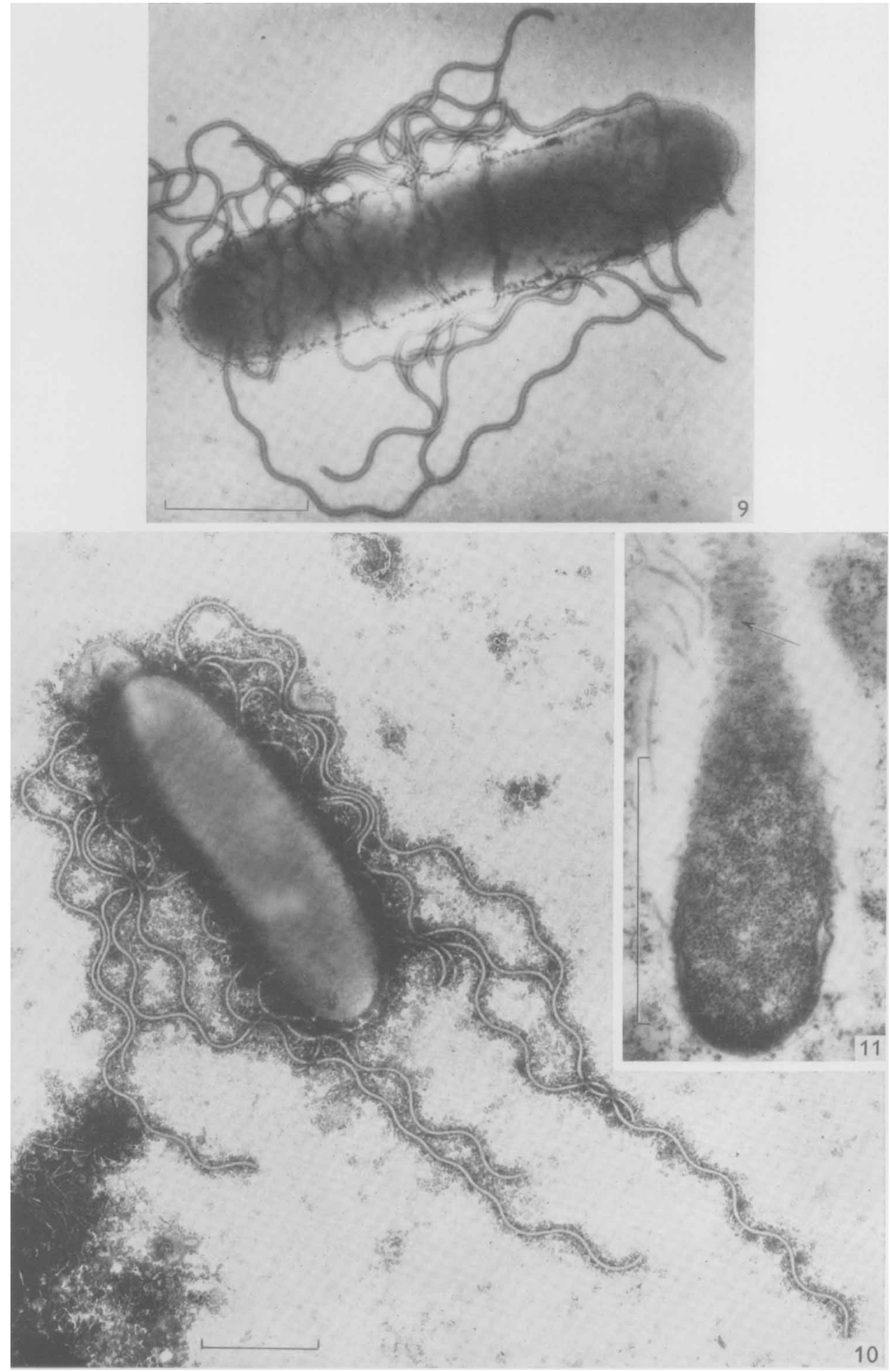

A. JURAND AND L. B. PREER 
Journal of General Microbiology, Vol. 54, No. 3

Plate 3
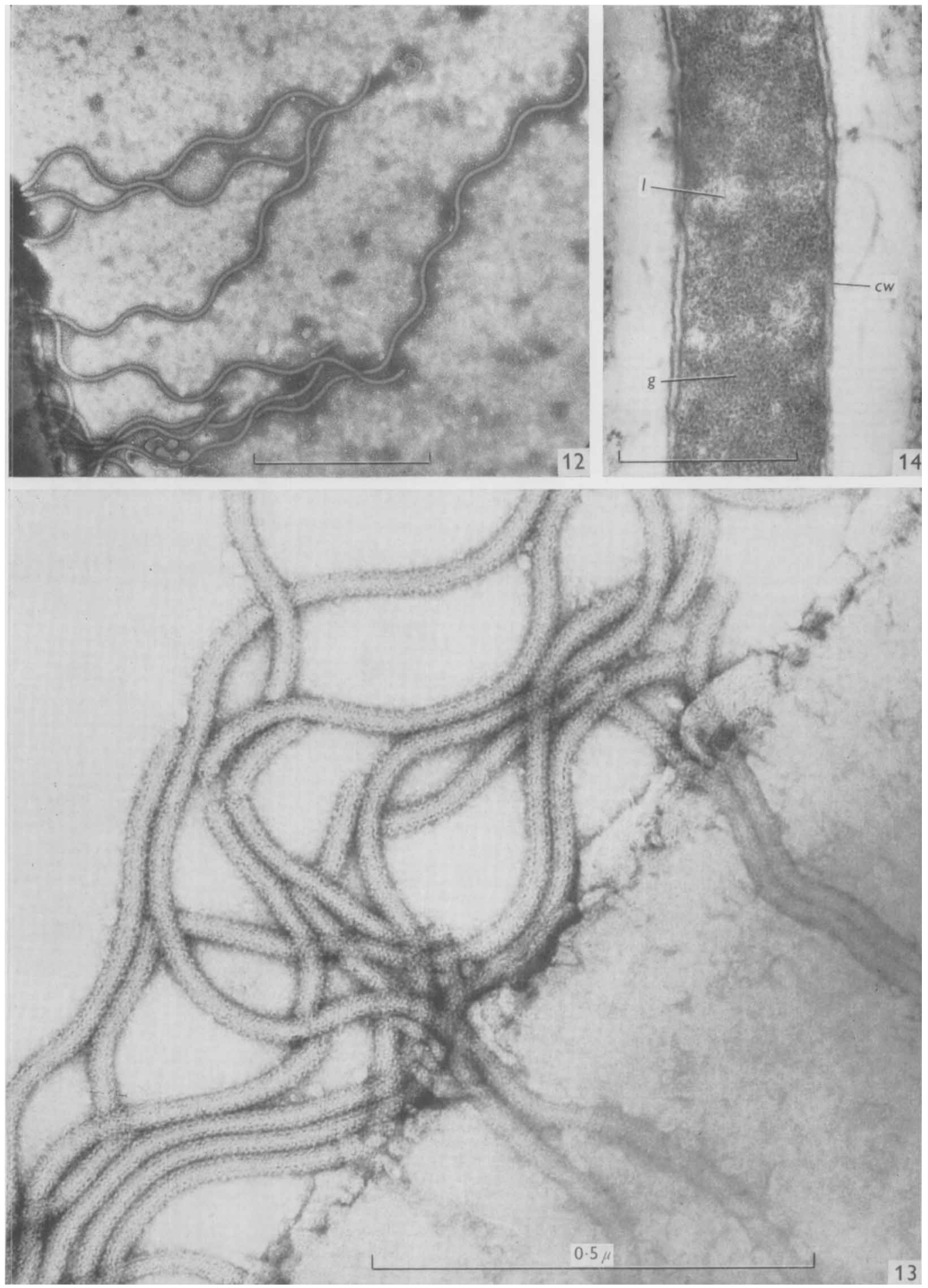

A. JURAND AND L. B. PREER 